\title{
Measurement technologies for permanent magnets
}

\author{
Stefan Möwius ${ }^{1}$, Nicolas Kropff ${ }^{1}$, Mircea Velicescu ${ }^{1}$ \\ ${ }^{1}$ BEC Gesellschaft für Produktmanagement $\mathrm{mbH}$, Zechenstraße 55, 47443 Moers, Germany
}

Permanent magnets have a broad application in many important fields in modern technology. They have become indispensable in the automotive, aerospace, acoustic, telecommunications, energy generation, and many more industries. Physically, a permanent magnet is a metastable system. Fluctuations in composition and processing parameters can cause fluctuations in magnetic properties. To obtain the optimal performance in their application, users require careful control of non-machined parts and of the finished machined magnets. In most cases, the measurement of the final control of the assembled magnetic systems must be performed. The aim of this paper is to review the most common measurement methods used for the magnetic properties of permanent magnets, to comment on their advantages and limitations, and to discuss the level of accuracy that they can achieve.

\section{Section: RESEARCH PAPER}

Keywords: Permanent Magnets, Hysteresis Graph, Helmholtz Coil

Citation: Stefan Möwius, Nicolas Kropff, Mircea Velicescu, Measurement technologies for permanent magnets, Acta IMEKO, vol. 7, no. 4, article 4, December 2018, identifier: IMEKO-ACTA-07 (2018)-04-04

Editor: Alexandru Salceanu, "Gheorghe Asachi" Technical University of lasi, Romania

Received March 26, 2018; In final form September 13, 2018; Published December 2018

Copyright: (๑ 2018 IMEKO. This is an open-access article distributed under the terms of the Creative Commons Attribution 3.0 License, which permits unrestricted use, distribution, and reproduction in any medium, provided the original author and source are credited

Corresponding author: Nicolas Kropff, e-mail: NKropff@bec-gmbh.de

\section{INTRODUCTION}

Permanent magnets are electrotechnical devices that can induce a magnetic flux in an air gap without the need for any other energy except that which is used for their magnetization. For the manufacture of permanent magnets, a magnetic material must combine optimal intrinsic hard magnetic properties with a suitable metallurgical microstructure. Useful magnetic materials are expected to have:

- a Curie temperature, $T_{\mathrm{C}}$, that is high enough to enable the operation of permanent magnets at temperatures of up to $200{ }^{\circ} \mathrm{C}$, without appreciable irreversible polarization losses,

- a high saturation polarization, $J_{\mathrm{s}}$, in the temperature range from $-40{ }^{\circ} \mathrm{C}$ to $200{ }^{\circ} \mathrm{C}$ to obtain high remanent polarization, $B_{\mathrm{r}}$, and to store the maximum magnetic energy density, $(B H)_{\max }$, and

- a strong uniaxial magneto-crystalline anisotropy, HA, to achieve high coercive field strength $H_{\mathrm{cJ}}$.

Due to the remanent polarization, the magnet induces a magnetic flux $\Phi$, which is the sum of the magnetic force lines through a defined cross-section in a heterogeneous magnetic field [2],

$\Phi=\int_{0}^{A} B$,

where $B=$ magnetic flux density or the magnetic induction and $A=$ cross-section. In the case of a homogenous magnetic field
$\Phi=B \times A$,

the characteristic lines of a permanent magnetic material are the hysteresis loops of the polarization $J(H)$, the intrinsic magnetic property of the material, and the induced flux density $B(H)$, plotted versus the magnetic field strength $H$. The $B(H)$ and $J(H)$ loops are correlated by the relationship

$B=J+\mu_{0} H$,

where $\mu_{0}=1.256 \cdot 10^{-6} \mathrm{Vs} / \mathrm{Am}$., the universal electromagnetic constant.

Users of permanent magnets are mainly interested in the second quadrant of the hysteresis curve, i.e., the demagnetization curves of the used permanent magnetic material as well as the load line of the permanent magnet, which depends on the specific magnet geometry. The demagnetization curve $J(H)$ shows how the external magnetic field changes the remanent polarization of the magnet, while the $B(H)$ demagnetization curve shows the magnet induction in a real air gap and is used especially for designing the magnet systems.

In terms of application, the users have a plethora of permanent magnetic materials at their disposal. The most commonly used hard magnetic materials that are available in mass production are AlNiCo; Hard Ferrite; and the permanent magnet materials based on rare earth alloys, namely $\mathrm{SmCo}_{5}$, $\mathrm{Sm}_{2} \mathrm{Co}_{17}$, and $\mathrm{NdFeB}$. The characteristic magnetic properties of the permanent magnetic materials are provided by manufacturers 
in their corresponding data sheets. The principal magnetic properties; the remanence $\mathrm{Br}$, the coercive forces of the polarization $H_{c J}$ and of the induction $H_{c B}$; and the maximum energy density $(\mathrm{BH})_{\max }$ are specified according to the typical experimental demagnetization curve $J(H)$ and to the resulting curve $B(H)$ according to Equation (2).

The properties of permanent magnets are generally very sensitive to their exact composition and the manufacturing parameters. Each type of permanent magnet that is mass manufactured fluctuates, leading in turn to fluctuations in their microstructure, and, further, to fluctuations in the magnet properties. The increasing use of the permanent magnets in essential applications involves implementing safety actions to assure their optimal performance. Users of permanent magnet technology are demanding quality control of the magnet properties from product development to serial production.

In this paper, the most typical magnetic measurement methods and adequate equipment for quality control and monitoring of permanent magnets from raw sintered parts to finished magnets are reviewed. Their advantages, disadvantages, and achieved accuracies are also discussed.

\section{MEASUREMENT METHODS FOR PERMANENT MAGNETS}

As mentioned above, the role of a permanent magnet is to induce a magnetic flux. Permanent magnet behavior, under the influence of temperature, opposite magnetic fields, and environmental conditions, is decisive in its performance in all applications. Users need a high magnetic flux that is created by a high remanence and high stability in opposite magnetic fields (especially at high) temperatures. The latter depends on the coercive force of the material and on its temperature coefficients and corrosion stability against the environment operating conditions, assured by the permanent magnet compositions and by surface protections.

For the characterization of the permanent magnets roughly two ways can be used: in a closed or in an open magnetic circuit. In a closed magnetic circuit, the magnet is in closed contact with magnetic conducting parts, for example between the poles of an electromagnet, and so the magnet flux is closed, without any airgaps between magnet and the ferrous parts. In an open circuit, the magnet is not in contact with ferrous parts, the measurement signals depend on the induced magnetic flux, i.e. is dependent on the magnet shape and thus on its load line. These signals must be converted by simulation models in signals corresponding to the closed circuit [1].

Different international and national standards are recommending measurement methods of the magnetic properties of permanent magnetic materials, for example [3-5]. They recommend the measurement in a closed circuit, commonly performed in a hysteresis graph. The highest accuracy is given only for magnets having a straight magnet axis and being produced with a constant cross section along the axis of magnetization. Furthermore, the magnet volume of the used samples must be not less than $1 \mathrm{~cm}^{3}$ and the smallest magnet dimension shall be $5 \mathrm{~mm}[3,4]$.

The performance of a magnetic application depends on the used permanent magnet material, as well as on their dimensions, air-gaps and on the soft magnetic components of the circuit. The number of new applications is continuously rising, very often leading to special and critical geometries and to requirements of very narrow magnetic tolerances. The aim of a measurement method is to determine the magnetic properties of magnets or of a magnetic system. Depending on the application, the magnet characterization means not imperatively to determine its principal magnetic parameters resulting from the characteristic lines $J(H)$ and / or $B(H)$. To ensure the high quality demanded by users, additional methods to control special magnetic properties are used.

In Table 1, an overview of the most typical measurement methods used for the characterization of the permanent magnets inspired from [6] and adjusted for this paper is presented. In this table the methods used for free magnets and those for magnets which have been assembled are separately mentioned.

\section{CHOICE OF MEASURING METHOD}

In choosing a measurement method, several factors must be considered. In terms of quality control in mass production, economic factors (e.g., the throughput of the pieces that must be characterized, the measurement time required per magnet, the relevant costs) and the required level of accuracy must be considered. For serial characterization, non-destructive measurement methods are more economical and are thus preferred. Depending on these requirements, the most suitable commercially available equipment must be used. For development and testing in laboratory conditions, equipment with improved accuracy increases the magnitude order of accuracy.

\section{A SHORT DESCRIPTION OF MEASUREMENT METHODS}

Besides the classical hysteresis graph, demagnetization curves can be traced by newly developed pulse-filed magnetometers for series measurement, while the Foner magnetometer is usually the preferred equipment for material research and development activities. For measurement of magnetic field strength and of magnetic induction, a Gaussmeter + Hall probe has been the universally preferred equipment for decades. The use of Helmholtz coils for measurement of remanence in open circuits and of the magnetic moment makes this equipment quite indispensable in laboratories and for the incoming controls of magnets. Furthermore, the homogeneity and 3D magnetic profiles can be controlled by 3D magnetic field mapping systems [7].

\subsection{Hysteresis Graph}

The working principles of a hysteresis graph according to the IEC's recommendation is shown in Figure 1 [3]. The measurement of the samples occurs in a closed circuit between two polar pieces with an adjustable air gap. The intensity of the magnet field is measured by a Hall sensor or by a coil, while a surrounding coil detects the flux density $B(H)$. Modern equipment uses only one compensated coil for the measurements of both parameters $J(H)$ and $B(H)$. It is additionally equipped with heating plates for tracing the demagnetization curves at high temperatures - up to $200{ }^{\circ} \mathrm{C}$.

\subsection{Pulse field magnetometer}

The first experiments on pulse fields for the measurement of high coercive forces were performed in 1988 by Grössinger [8]. The intensive research and development efforts of a European group [9] have shown that this method can be applied to the industrial characterization of permanent magnets. A diagram of the working principles of the pulse field measurement method following [10] is presented in Figure 2. A sinusoidal magnetic 


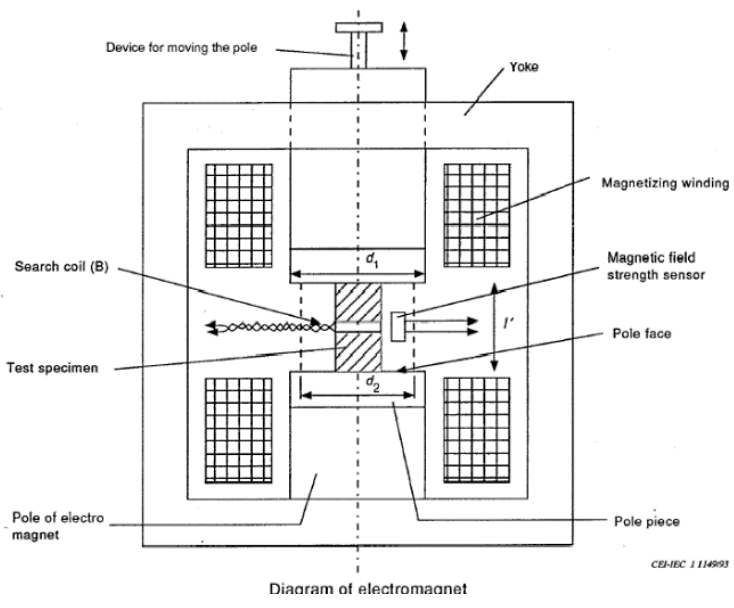

Figure 1: Diagram of the working principles of a hysteresis graph.

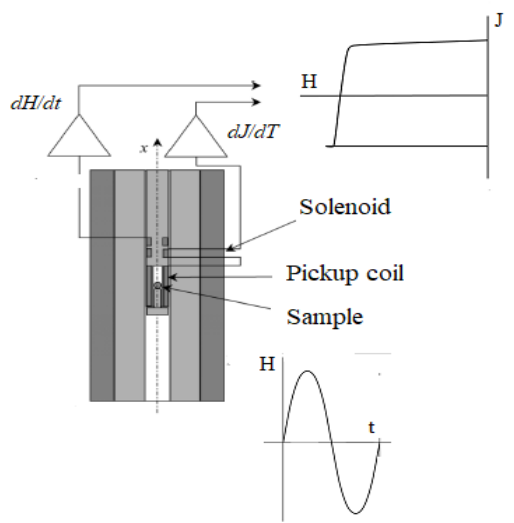

Figure 2. Diagram of the working principles of a pulse field magnetometer.

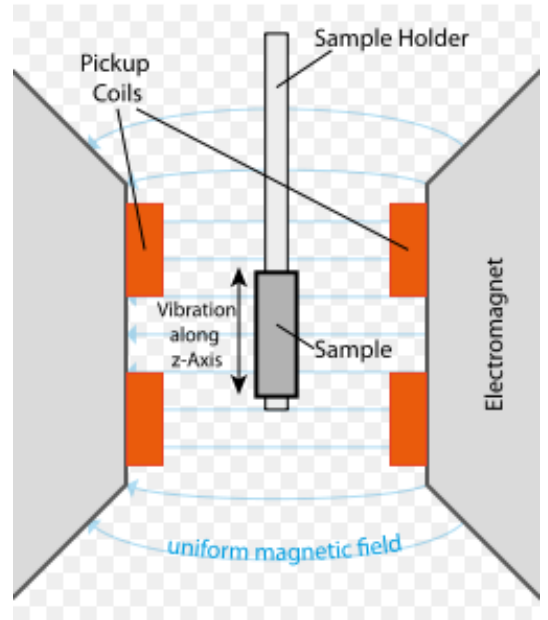

Figure 3. Diagram of the working principles of a Foner magnetometer.

damped field is generated by discharging a bank of capacitors trough the solenoid. The magnetic polarization of the sample is measured by pickup coils, while a Hall probe or another field sensor measures the field strength, allowing for the tracing of the $\mathrm{J}(\mathrm{H})$ curve.

This method has several important advantages, namely the high magnetizing field strength, which ranges between 7 and 15 $\mathrm{T}$, and the facility to measure, in a non-destructive way, samples with arbitrary geometric forms, e.g., arc segments, a bread shape, and diametrical-oriented cylinders. Even though this method has not been recognized by an international norm until now, this equipment is produced serially today and is already used on a large scale for research and development as well as serial quality control. The measurement can be performed at temperatures of up to $200^{\circ} \mathrm{C}$. Specials facilities have been developed to perform measurements in the low temperature range of $20-40{ }^{\circ} \mathrm{C}$ [11].

Table 1: Measurement methods used for the characterization of permanent magnets

\begin{tabular}{|c|c|c|c|c|}
\hline Measurement Method & Parameters & Units & Equipment & Accuracy $[\%]$ \\
\hline \multicolumn{5}{|c|}{ Measurement of free magnets } \\
\hline \multirow{3}{*}{$\begin{array}{l}\text { Demagnetization } \\
\text { Curves }\end{array}$} & \multirow{3}{*}{$J(H), B(H)$} & \multirow{3}{*}{$\mathrm{T}, \mathrm{kA} / \mathrm{m}$} & Hysteresis Graph & $(+/-) 1$ \\
\hline & & & $\begin{array}{l}\text { Pulse Field } \\
\text { Magnetometer }\end{array}$ & $(+/-) 1$ \\
\hline & & & $\begin{array}{l}\text { Foner Magnetometer } \\
(\text { VSM) }\end{array}$ & $(+/-) 0.5$ \\
\hline $\begin{array}{l}\text { Remanence in open } \\
\text { circuit }\end{array}$ & $J_{\mathrm{oC}}$ & $\mathrm{T}$ & Helmholtz Coil & $(+/-) 1$ \\
\hline Magnetic moment & $M_{\mathrm{m}}$ & Vs cm & Helmholtz Coil & $(+/-) 1$ \\
\hline \multirow{2}{*}{ Angle deviations } & \multirow{2}{*}{$J$} & \multirow{2}{*}{ Degree } & Helmholtz Coil & $(+/-) 0.5^{\circ}$ \\
\hline & & & 3D Mapper & $(+/-) 0.5^{\circ}$ \\
\hline \multirow[t]{2}{*}{$H(B)$ in a test point } & \multirow[t]{2}{*}{$B$} & \multirow[t]{2}{*}{$\mathrm{T}$} & $\begin{array}{l}\text { Gauss Meter / Hall } \\
\text { Probe }\end{array}$ & $(+/-) 1$ \\
\hline & & & 3D Mapper & $(+/-) 1$ \\
\hline Surrounding coil & $\Phi$ & Vs cm & Coil and Fluxmeter & $(+/-) 0.5$ \\
\hline Holding force & $F$ & $\mathrm{~N}$ & Dynamometer & $2-5$ \\
\hline \multicolumn{5}{|c|}{ Measurement of assembled systems, e.g. rotors } \\
\hline$H(B)$ in air gap & $B$ & $\mathrm{~T}$ & $\begin{array}{l}\text { Gaussmeter / Hall } \\
\text { Probe }\end{array}$ & $1-2$ \\
\hline EMF & $U_{\text {eff }}$ & $\mathrm{V}$ & $\begin{array}{l}\text { Motor Coil / } \\
\text { Voltmeter } \\
\end{array}$ & 1 \\
\hline
\end{tabular}


In terms of electrical conducting metallic magnets like SmCo or $\mathrm{NdFeB}$, this method induces eddy currents. This phenomenon influences the value of the remanent polarization. To correct the measured data, modeling calculations have been developed.

\subsection{Foner or vibrating sample magnetometer (VSM)}

The vibrating sample magnetometer (VSM) was developed in the 1950s by Foner [12]; therefore, it is also known as the Foner magnetometer. Figure 3 shows the working principles of this method. Initially, a loudspeaker membrane [13] was used for the vibration of a sample holder and thus of the sample. An electromagnet creates the variable external magnetic field. Its strength is measured by a Hall sensor. Other sources of a variable magnetic field have been developed, e.g., superconducting solenoids [14], Bitter coils [15], and even permanent magnet assembled systems $[16,17]$.

With a vertical vibration of $80 \mathrm{~Hz}$ [12], the sample induces a signal that is proportionate to its magnetic polarization in the pickup coil. The samples are measured in open circuit and the signal requires corrections caused by the shearing of the demagnetization factor of the polarization.

For a long time, the Foner magnetometer was the most common measurement method for soft and hard magnetic materials in the temperature range of $2-1000 \mathrm{~K}$. The sample size is limited to any $\mathrm{mm}^{3}$. The overall measurement accuracy depends on the measurement accuracy of the sample volume. Nowadays, the VSM method is mostly used in research centers.

\subsection{Helmholtz coil (HHC)}

The Helmholtz Coil (HHC) was invented at the end of the $19^{\text {th }}$ century to generate small, highly uniform magnetic fields. It consists of two identical coils connected in a series, placed symmetrically along an axis, and separated by a distance that is equal to their radii. Its initial idea has been reversed. For magnetic measurements, the coils are used as flux-sensing coils to accurately measure magnetization in open circuit [17]. Figure 4 shows a typical configuration of the HHC.

By connecting it with an electronic fluxmeter, the HHC allows for the measurement of the remanence in open circuit, of the magnetic moment, and of the deviation angle between the magnetic and geometric axes with a high level of accuracy. Such measurement can be performed for serial production. To collect data, different calculations and integrations must be conducted. The most important parameters are the coil constant, the demagnetization factor, and the volume of the analyzed magnet. Based on the easily comprehensible measurement process, the magnet is inserted between both coils and the measured field is reset to zero. Thereafter, the magnet is rotated $180^{\circ}$. By turning

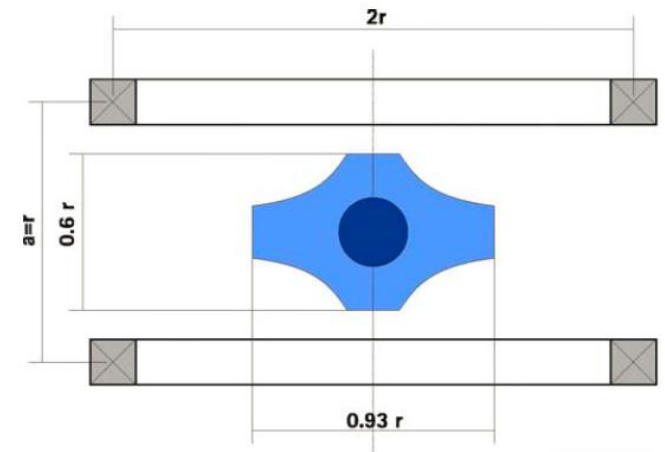

Figure 4. A typical configuration of the Helmholtz coil according to [18]. the magnet inside the coils, the magnetic field is turned $180^{\circ}$, too, and as a result, the HHC can measure the signal entirely [18].

The HHC has become indispensable equipment for incoming control of permanent magnets and for laboratory tests.

\subsection{Hall Probe}

The Hall Probe, Figure 5, is used to measure magnetic field strength or flux density. It is also deployed as a sensor device in various applications. The inherent Hall Generator consists of a semiconducting material and is passed by a control current. If a magnetic field is applied perpendicularly to the measurement surface of the Hall Probe, the Lorentz Force acts on the moving charge carriers passing the generator. The Lorentz Force is expressed as:

$F_{L}=q(v \times B)$,

where $q$ is the particle charge, $v$ is the particle velocity, and $B$ is the magnetic flux density.

The resulting force pushes the charge carriers sideways, thus enabling the measurement of the corresponding Hall Voltage $\mathrm{U}_{\mathrm{H}}$, expressed as:

$U_{H}=R_{H} \times \frac{I \times B}{d}$,

where $R_{H}$ is the Hall constant, $I$ is the passing control current, $B$ is the magnetic flux density, and $d$ is the thickness of the Hall Generator [19, 20].

While carrying out a measurement using a Hall Probes, the user must consider the sensitivity of the measuring device. The volume of the Hall Generator itself is crucial, as indicated in Equation (5). Flux density $B$ is a temperature-dependent parameter [21].

\subsection{Gaussmeter}

A Gaussmeter combined with a Hall Probe is a universally preferred equipment type that is used for measuring magnetic flux density $B$ or magnetic field strength $H$ statically, in a desired location or space. Both parameters can be determined in air gaps, too, e.g., in engines or loudspeakers.

A Gaussmeter is a suitable device for the quality control of serial produced magnets as it carries out measurements of magnetic field strength at a definite point and at a definite distance from the magnet surface. The "quality" of the tested magnet is evaluated comparatively with the flux density of a calibrated reference magnet.

The resolution and the accuracy of a Hall Gaussmeter depend on the size and the properties of the Hall sensor [22]. Newly developed Hall Gaussmeters that use miniaturized horizontal

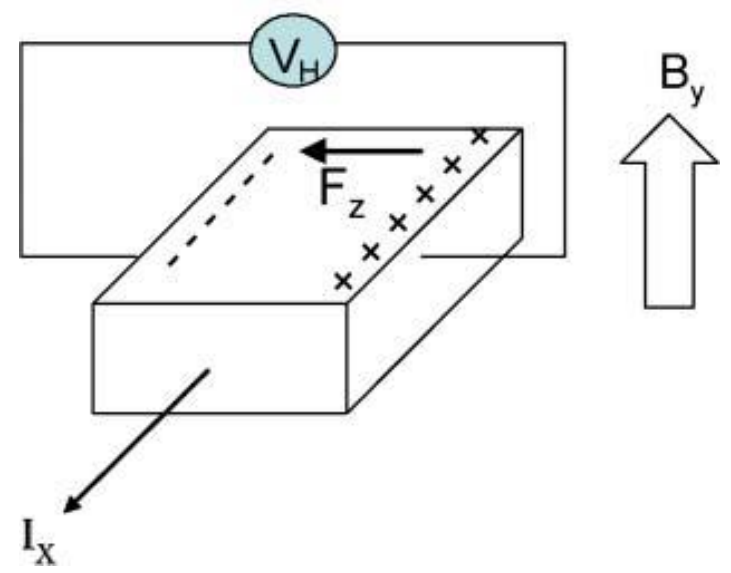

Figure 5. Schematic configuration of a Hall probe according to [19]. 


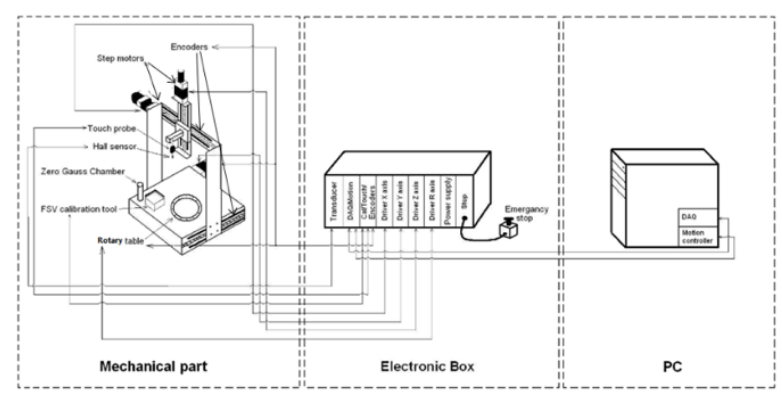

Figure 6. Block diagram of a commercially available magnetic mapper, reprinted with permission of SENIS AG.

and vertical Hall devices, the so-called integrated 3-axis Hall Probes, achieve extremely high resolution and accuracy [23].

\subsection{D Mapper}

In many permanent magnet applications, the magnetic field distribution over an axis, area, or volume is essential in the magnetic system. This parameter is concerned with rotation and linear motors, especially magnets assembled in positioning sensor systems. Control of the magnetic field near and around a magnet can be performed using a magnetic field mapper. A block diagram of a commercially available $3 \mathrm{D}$ mapper is presented in [24]. The main parts of such a mapper are the moving platform; the Hall Probe; the electronic box (with digital processing); the motor drives and power supply; and a PC and software for collection of the measured data and the visualization and analysis thereof, see Figure 6.

One of the most important benefits of such 3D mappers is the measurement of complete systems and the visualization of all magnetic components. Thereby, it is possible to evaluate, e.g., the field distribution in a magnetic system or to analyze the magnetic pole symmetry in a multiple magnet. All these measurements are performed with a 3-axis Hall Probe. Besides the magnetic measurements, such measurement equipment can be used with other sensors, e.g., for detecting cracks within the magnet body or measuring the dimensions of the analyzed samples. The sensors can be changed by using a plug-and-play system, and the measured data can be combined in one system for detailed analysis.

The so-called defectoscope can identify cracks and inhomogeneities within magnetized and unmagnetized magnets by using an eddy current measurement probe. The probe measures the eddy current distribution and creates a visual scan of the surface to show the defects $[25,26]$.

\section{MEASUREMENT RESULTS}

All of the measurement technologies explained above have one thing in common: they are used to examine the magnetic properties of a magnet or a magnetic system in an open or closed circuit. The compliance of the special magnetic properties with quality control requirements is important for magnet suppliers and essential for customer. The choice of measurement technology made is according to these parameters. Generally, the measured results can be divided into two groups.

The first results group is for mass production, where the parts are only differentiated between "good" and "not good" items and are sorted accordingly. Therefore, there is no further evaluation of the collected data. Such measurement methods are highly cost-sensitive and require a high output of analyzed permanent magnets to be economically viable.

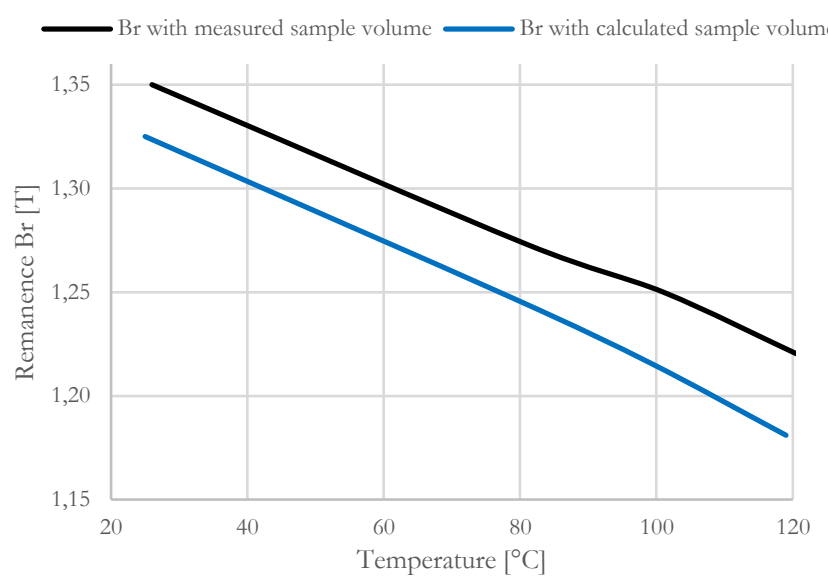

Figure 7. Metis HyMPulse measurement of $\mathrm{Br}$ over increasing temperature $T$ with the measured and calculated sample volume, performed in 2017 at the BEC laboratory.

The second results group is for research and development. The collected data is analyzed in a highly detailed way, as it is required for further improvement of and modifications to the materials or application.

In addition to the influence of different measurement technologies, the results obtained by magnetic measurements can be influenced by the interpretation and consideration of the sample's intrinsic parameters as well.

Figure 7 shows the measured magnetic remanence $\mathrm{Br}$ over an increasing temperature $\mathrm{T}$, obtained by applying the Metis HyMPulse measurement. Two measurements of the same magnet sample indicate significantly varying results, such variance depending on the underlying sample volume. The curve for the sample measurement with the calculated sample volume (based on the technical drawing) shows a reduced magnetic remanence compared to the measurement curve with the precisely measured sample volume (volume measured by using the Archimedes method). As the investigated sample was produced at the upper limit of the drawing-specified dimensions, the measured sample volume turned out larger than the calculated sample volume. Based on the dependency of the magnetic remanence $\mathrm{Br}$ on volume, different measurement results were obtained.

\section{CONCLUSION}

We reviewed the most commonly used methods for magnetically characterizing permanent magnets in laboratories, e.g., in their development state, and for quality control in the serial production thereof.

Different measurement methods and suitable measurement equipment are commercially available for most required characterizations related to the magnetic properties and magnetic behavior of permanent magnets.

By choosing the most adequate measuring method, unnecessary controlling costs can be avoided.

The combined use of multiple measurement methods can contribute to the improvement of knowledge concerning the behavior of permanent magnets and thus to the achievement of good quality assurance. The BEC laboratory is equipped according to this regard. 


\section{ACKNOWLEDGMENT}

The authors wish to thank Dr. Dragana Popovic Renella for a helpful discussion during preparation of this paper.

\section{REFERENCES}

[1] B. Grieb, Hartmagnetische Werkstoffe. Magnet werkstoffe für technische Anwendungen. Essen, Haus der Technik, 2016.

[2] E. Steingroever, Magnetic Measuring Techniques,

[3] IEC International Standard 60404 Part 5: Permanent magnet (magnetically hard) materials - Methods of measurement of magnetic properties, 2015.

[4] DIN EN 60404 - 5VDE 0354-5: 2016-02 "Magnetische Werkstoffe".

[5] MMPA Standard 0100-00: "Standard specifications for permanent magnet materials".

[6] K. Kuntze, Chapter 6, in TAE Band 672, "Dauermagnete", Expert Verlag, 2015

[7] S. R. Trout, IEEE Trans. Mag. 24(4) (1988), p. 2108.

[8] R. Grössinger, C. Gigler, A. Keresztes, IEEE Trans. Mag. 24(2) (1988), p. 970

[9] R. Cornelius, J. Dudding, R. Grössinger, B. EnzbergMahlke, W. Fernengel, M. P. Knell, M. Küpferling, M. Taraba, J. C. Toussaint, A. Wimmer, and D. Edwards, IEEE Tans. Mahn. 38(5) (2002), p. 2462.

[10] F. Fiorillo, C. Beatrice, O. Bottauscio, E. Patroi, IEEE Trans. Mag. 43(7) (2007), p. 3159.

[11] L. Van Bockstal, Technical Presentation HyPulse Technology, 2017.
[12] S. Foner, Rev. Sci. Instrum. 30 (1959) p. 548

[13] D. Feldmann, R. P. Hunt, Z.Instr. 72(11) (1964), p. 313.

[14] S. Foner, J. Appl. Phys. 79 (1996) p. 4740.

[15] D. K. Das, W. J. Harrold, IEEE Trans. Mag. 17 (1981) p. 281.

[16] O. Cugat, P. Hansen, J. M. D. Coey, IEEE Trans. Mag. 30 (1994) p. 4602.

[17] J. M. D. Coey, J. Magn. Magn. Mat. 248 (2002) p. 441.

[18] Brockhaus - Messtechnik Data sheet "measurements using the Helmholtz coil"

[19] S. Johnstone, The Analyst 133 (2008) pp. 293-296.

[20] W. Zhenxing, S. Mehrdad, S., M. Otto, D. Schall, D. Neumaier, Nanoscale 8 (2016) pp. 7683-7687.

[21] K. Kuntze, Chapter 3, in TAE Band 672, "Dauermagnete", Expert Verlag, 2015.

[22] R. S. Popovic (editor), Hall Effect Devices, 200, CRC Press, Taylor \& Francis Group.

[23] D. Popovic Renella, S. Dimitrijevic, S. Spasic, R. S. Popovic, $20^{\text {th }}$ IMEKO Int. Symp. 2014, Italy.

[24] S. Spasic, Magnetics, Business \& Technology, Spring (2015) p. 16

[25] Y. G. Guo; J. G. Zhu, Z. W. Lin, J. J. Zhong, Measurement and modeling of core losses of soft magnetic composites under 3-D magnetic excitations in rotating motors, IEEE Trans. Mag. 41(1) (2005).

[26] W. Xin, K. Ding. Magnetic measurement method on structure fatigue damage based on the material magnetic characteristics, Chinese Journal of Scientific Instrument 38(6) (2017) pp. 1474-1481. 